Théologiques

Théologiques

\title{
Le discernement spirituel dans le christianisme ancien
}

Le problème de la décision

\section{Fabrizio Vecoli}

Volume 22, numéro 2, 2014

Le discernement spirituel

URI : https://id.erudit.org/iderudit/1035687ar

DOI : https://doi.org/10.7202/1035687ar

Aller au sommaire du numéro

\section{Éditeur(s)}

Faculté de théologie et de sciences des religions, Université de Montréal

ISSN

1188-7109 (imprimé)

1492-1413 (numérique)

Découvrir la revue

Citer cet article

Vecoli, F. (2014). Le discernement spirituel dans le christianisme ancien : le problème de la décision. Théologiques, 22(2), 69-97.

https://doi.org/10.7202/1035687ar
Résumé de l'article

L'article analyse le discernement spirituel dans le christianisme ancien. Le but est de comprendre l'évolution conceptuelle et les fonctions religieuses de cette capacité considérée comme divine par les auteurs qui en développent la théorie. Après une première mention dans les épîtres de Paul, on constate un vide dans la théologie des premiers siècles. C'est donc seulement au $\mathrm{IV}^{\mathrm{e}}$ siècle, avec l'essor du monachisme, que le discernement spirituel acquiert toute son importance. Il représente l'instrument cognitif qui rend possible le processus décisionnel dans une situation d'ignorance : ce don permet de voir les mécanismes cachés du réel afin de toujours prendre la bonne décision. D'abord utilisé individuellement comme guide dans la pratique de l'ascèse, il finit par devenir l'emblème d'une autorité charismatique puissante et le signe de reconnaissance de la sainteté. 


\title{
Le discernement spirituel dans le christianisme ancien
}

\author{
Le problème de la décision
}

\author{
Fabrizio VeCOLI" \\ Histoire du christianisme \\ Université de Montréal (Canada)
}

\section{Méthode}

Le discernement spirituel peut être observé dans sa dimension de critère présidant à l'action du croyant dans le monde ou — avec une tout autre perspective - au niveau de l'élaboration théorique qui explique la nature de ce critère, en détaille les fonctions et en légitime l'existence ${ }^{1}$. S'il est donc vrai qu'il occupe à la fois la sphère très concrète de la pratique sociale et celle plus éthérée de la réflexion théologique - et qu'il fait montre par suite d'une certaine amphibologie taxinomique -, il apparaît néanmoins qu'il s'agit d'un objet susceptible d'être étudié dans le cadre d'une discipline dénommée, à la suite de Hegel, histoire des concepts (Begriffsgeschichte) (Meier 1971). Ainsi, la chose qui dans nos sources est fréquemment classée sous la catégorie du charisme divin peut être traitée comme un concept. Aboutissement d'une évolution langagière originale qui a mené à re-sémantiser un terme ancien afin de nommer ce qui se présentait comme une compétence émergente parmi les suivants de Jésus, le discernement fait montre d'un remarquable niveau d'abstraction dans le

* Fabrizio Vecoli est professeur adjoint à la Faculté de théologie et sciences des religions de l'Université de Montréal. Son travail de recherche se concentre actuellement sur le concept de discernement dans l'histoire du christianisme. Parmi ses œuvres, la monographie (2007) Il sole e il fango. Puro e impuro tra i Padri del deserto, Rome, Edizioni di Storia e Letteratura reflète son intérêt pour l'histoire du monachisme ancien.

1. Je tiens à remercier Louis-Charles Fauteux pour son travail d'auxiliaire de recherche dans le cadre de mes études sur le discernement spirituel. 
traitement que lui font subir les textes. Et c'est surtout à ce niveau qu'il nous intéresse, car "face au réel, l'homme ne peut pas ne pas sémantiser, et cette sémantisation détermine que désormais pour lui les choses n'existent que conçues» (Giot-Schotte 1999, 76). Il est par suite légitime d'appréhender le discernement comme le résultat d'une opération réfléchie de représentation de ce que cette capacité est ou devrait être à l'intérieur d'un contexte culturel particulier, dans notre cas le christianisme ancien. On pourrait affirmer que le concept, en tant que produit de représentation, se développe là où l'empirique expérientiel rencontre le spéculatif ou - pour mieux dire - là où le réel devient discours ${ }^{2}$. En ce sens, on ne saurait séparer le concept du milieu social et culturel d'où il est issu, car on ne peut nier que c'est bien le contexte qui en détermine à chaque fois la déclinaison spécifique, c'est-à-dire la variante distinctive parmi ses innombrables récurrences à travers les siècles. En effet, de discernement on parlera tout le long de l'histoire du christianisme. Se situant donc dans la temporalité (Koselleck 1997), il est de tous les points de vue un objet historique. Pourtant, remettre en contexte la réflexion sur le discernement contenue dans nos sources ne suffit pas. On se heurte à une difficulté inhérente à sa nature, à la fois abstraite et concrète, qui rend malaisé de déterminer à quelle temporalité il faut le référer: les catégories de diachronie ou de synchronie s'avèrent trop approximatives face à un croisement inextricable de cadences qui sont, d'une part, celle de l'affirmation du concept comme terme pour la réflexion doctrinale et, d'autre part, celle de la longue gestation des pratiques auxquelles il renvoie. C'est un peu comme si, pour reprendre les catégories de Braudel (Braudel 1949), nous avions affaire - en référence à un même objet historique — à la fois aux temps événementiel, conjoncturel et de longue durée. On le constate d'ailleurs en lisant les textes: le discernement pris à un moment précis de son histoire fait référence, dans le discours qui en parle, à un contexte de pratiques se développant à un rythme temporel différent. Ce mot, entré dans le lexique théologique, met en tension le passé de son émergence graduelle et le futur des attentes qu'il exprime, et - j'ajouterais - des développements qu'il conditionne ( All sociopolitical concepts encounter a temporal tension which assigns the past and the future in a new way ", Koselleck 1997b, 20). On ne peut donc qu'admettre, encore une fois, l'existence d'un écart problématique entre le fait historique et le mot qui

2. Nous adhérons à la position de Guilhaumou (2006, 181-221), qui affirme la nécessité d'une connexion entre le discours et un réel extérieur à lui. 
le dit (et "crée» donc l'évènement). Les deux se meuvent sur des échelles temporelles décalées, ce qui rend, du moins pour Koselleck (1997, 105), l'histoire «totale» impossible. Bien entendu, cela est particulièrement vrai quand il s'agit d'un concept théologique. Il y a donc, entre évènement et discours, un espacement qui n'est pas sans nous évoquer la relation proposée par Pierre Bourdieu ${ }^{3}$ entre la révolution politique et la révolution symbolique: l'interprétation du discernement donnée par les théoriciens du monachisme, qui modifie radicalement celle des auteurs précédents et lui confère par ce fait même une identité spécifique, relève d'une certaine manière de la révolution symbolique ${ }^{4}$. Pourtant celle-ci n'a certes pu se mettre en place sans les mutations souterraines qui l'ont préparée. De façon réciproque, la «révolution politique » qui l'a précédée n'a pu trouver son véritable accomplissement et acquérir sa visibilité sans l'explicitation du langage qui l'a dite.

Dans cet état des choses, il semble profitable de suivre la démarche qui fut jadis celle de Michel De Certeau dans son étude du phénomène mystique $^{5}$, un objet qui - de par son statut relevant d'une élaboration conceptuelle - entretient une relation de proximité avec le discernement. Il apparaît ainsi opportun de commencer par fixer le regard là où — semblet-il - a lieu une première élaboration formelle du discours sur le discernement, soit en ce lieu de l'histoire chrétienne où le mot et la chose se sont rencontrés dans le cadre d'un discours conscient. Or, c'est précisément dans les textes monastiques du $\mathrm{IV}^{\mathrm{e}}-\mathrm{v}^{\mathrm{e}}$ siècles que l'on trouve une attention particulière à ce charisme divin, au point où l'on peut parler — en ce qui le concerne - d'une véritable doctrine. Il y a dans ce choix un certain

3. «La relation qui s'établit entre la révolution politique et la révolution symbolique n'est pas symétrique. S’il n'est sans doute pas de révolution symbolique qui ne suppose une révolution politique, la révolution politique ne suffit pas, par soi, à produire la révolution symbolique qui est nécessaire pour lui donner un langage adéquat, condition d'un plein accomplissement» (Bourdieu 1971, 334).

4. Dominique Bertrand le constante (sans pour autant appliquer le filtre sociologique de Bourdieu): «Il a fallu que le monachisme devienne une force culturelle dans l'Église et le monde ambiant pour que le discernement des esprits se remplisse d'un contenu précis et qui, sauf effacements temporaires, ne variera plus » (Bertrand 2003b, 375).

5. «Mieux vaut donc s'en tenir provisoirement à ce qui se passe dans les textes où "mystique" figure comme l'index de leur statut [...]", l'important étant de "déterminer ce qui survient dans le champ que découpe un nom propre ["mystique"] et où s'effectue un travail soumis à un ensemble de règles. [...] Au départ de l'analyse, il y a donc l'isolement d'une unité "mystique" dans le système de différenciation des discours qui articule un nouvel espace de savoir» (De Certeau 1995, 28). 
déplacement par rapport à une logique chronologique traditionnelle, puisque notre centre de gravité ne se situe pas aux origines du phénomène, vagues et incertaines (sans compter tous les problèmes que soulève la catégorie d' "origine" en histoire), mais au moment où le terme auquel nous faisons référence se revêt pour la première fois d'une signification déterminée à l'intérieur d'une réflexion articulée.

\section{Terminologie}

Le haut niveau d'élaboration théorique affiché par nos auteurs monastiques lorsqu'ils parlent du discernement n'enlève pas le fait qu'ils utilisent un mot dont nous devons retracer l'histoire, afin de mieux le comprendre.

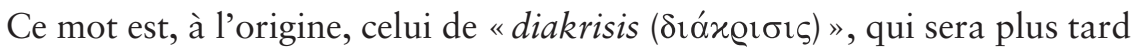
traduit par "discretio» en latin. Le lexique de Liddell-Scott (Liddell et al. 1996, 399) nous indique que l'usage classique de ce terme grec identifiait deux sphères sémantiques principales, soit l'acte de la division/séparation et le jugement qui est donné dans un cadre formel (concours, procès, diagnostic médical). Les deux acceptions ne sont pas éloignées, puisque pour trancher le juge doit effectivement déterminer - justement en les séparant - le vrai et le faux, le juste et l'injuste, le bon et le mauvais, le beau et le laid; et, sur la base de cette dichotomie, il doit ensuite prendre une décision (cet élément revient dans toutes les variantes de cette deuxième acception). Dans la Bible, l'emploi de ce terme insiste sur l'idée du choix à faire après examen - entre des alternatives différentes, voire opposées (Rich 2007, 1-38). La particularité de ce choix consiste dans le fait qu'il présuppose une identification préalable des options, qui toutefois maintiennent une part d'obscurité : même pour le prophète ou le saint la voie de Dieu comme celle du péché demeurent, dans les tréfonds de leur essence, mystérieuses (Guillet 1957).

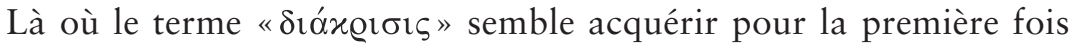
une connotation technique, bien qu'encore difficile à cerner, c'est dans la Première Épître aux Corinthiens (12, 4-10). Paul y énumère les charismes octroyés par l'Esprit, qui diversifient le corps des croyants à l'intérieur du tout organique de la communauté: c'est parmi ceux-ci que l'on trouve

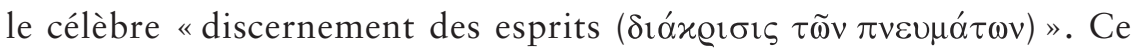
qu'il faut bien saisir de la nature de ces dons spirituels chez Paul (Ducros 1953, 503-507; Lampe 1968, 1518-1519; Martin 1982, 1015-1088; Conzelmann 1988, 606-616; Berger 1998, 1886-1889; Merkel H. et Wallis R. 1999, 402-404), c'est qu'elle souligne la spécificité de chaque 
vocation ${ }^{6}$ dans le contexte de l'assemblée des fidèles; l'individualité du croyant qui s'en trouve appuyée ne se résout aucunement en une quelconque autonomie, mais exprime plutôt la partialité d'un pouvoir particulier qui se doit de fonctionner en syntonie avec les autres. En effet, le charisme paulinien n'est pas et ne rend pas autonome dans ce que Bourdieu appelait la «gestion des bien de salut» (Bourdieu 1971, 304). Au contraire, il exprime le caractère propre de chaque fidèle, qui se distingue à la façon d'une tesselle dont la tonalité diffère certes des autres mais n'a de sens qu'en s'insérant dans l'ensemble harmonique de la mosaïque. Selon cette conception, le charisme paulinien - discernement ou autre - est certes nécessaire au fonctionnement de l'Église mais n'est pas fonctionnel au salut. Pour en venir plus spécifiquement au discernement des esprits, ce don spirituel permet de reconnaître la nature (utile ou non à la communauté) d'une inspiration surnaturelle. De toute évidence, la distinction de ces «esprits » n'est pas immédiate et nécessite par conséquent d'une assistance surhumaine. Il est difficile d'en dire plus en raison de la concision de Paul à ce sujet (la formule "discernement des esprits» est un hapax dans son œuvre). C'est un obstacle qu'on préférera ne pas contourner - bien que certains l'aient fait - en établissant une

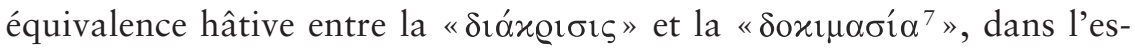
poir de préciser la première ${ }^{8}$. Ce seront des auteurs plus tardifs qui développeront la réflexion sur le discernement.

\section{La lacune du christianisme primitif}

L'évidence d'une lacune dans le christianisme des premiers siècles, quant à la réflexion sur le discernement spirituel, a déjà été relevée (Lienhard 1982, 519; Monaci 2009, 9): on est même allé jusqu'à parler d'un «vide théorique» (Bertrand 2003a, 969). En effet, avant l'essor du monachisme,

6. Elle-même expression de la grâce (en grec le lien est étymologiquement évident: grâce

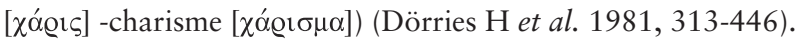

7. Autre notion présente dans le corpus paulinien: l'acte de mettre à l'épreuve.

8. D'ailleurs, certains auteurs ne s'embarrassent nullement de traduire le verbe

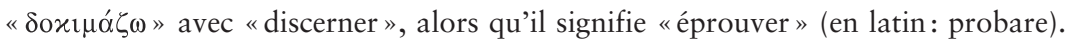
Le discernement présuppose un savoir qui précède la vérification et ne requiert aucune mutation dans les qualités de celui qui l'exerce; au contraire, la «mise à l'épreuve» produit une connaissance successive à une vérification qui, par ailleurs, est aussi un raffinement (donc une amélioration/purification). En ce sens, la monographie de Gérard Therrien (1973) est un exemple frappant: à quelques exceptions

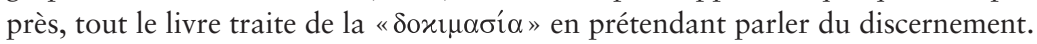


Origène (185-253) est le seul auteur à traiter de la question du discernement de façon quelque peu systématique. À vrai dire, au $\mathrm{II}^{\mathrm{e}}$ siècle, Hermas avait fourni quelques détails sur les moyens d'identification des esprits (Pasteur, Préceptes 6,2,1-4), sans toutefois jamais nommer explicitement le charisme paulinien. Il est néanmoins intéressant de relever que sa manière d'aborder la question s'avère tout à fait compatible avec ce que suggérait en elle-même la mention d'un charisme du "discernement des esprits» chez Paul: c'est le problème d'une opacité à éclaircir. Hermas s'interroge en effet sur la façon de reconnaître l'ange de justice par rapport à celui de l'iniquité, "car les deux habitent avec moi». Il énonce ainsi de façon explicite le problème présent in nuce chez l'apôtre des gentils: les "esprits» ne se distinguent pas aisément. Il faut par ailleurs retenir, dans le texte du Pasteur, deux éléments de nouveauté destinés à revenir par la suite. Tout d'abord, ce qui se configure comme un don de l'Esprit chez Paul prend ici l'allure d'une technique fondée sur l'analyse psychologique, où les émotions suscitées par la manifestation des esprits permettent de distinguer ces derniers (calme $=$ divin ; agitation $=$ démoniaque $)$. En deuxième lieu, nous assistons chez Hermas (et surtout dans la relecture de celui-ci par Origène) à la soudure du thème paulinien des esprits/inspirations dans la communauté avec celui, plus ancien, des deux voies du salut et de la damnation (et des deux esprits qui président à celles-ci): des textes de Qumran (Règle de la communauté 3,13-4,26) aux théologiens du christianisme ${ }^{9}$, la transmission de ce thème dualiste (Moreschini et Norelli 2000, 162, Attridge 2000, 204) s'avère lourde de conséquences pour le développement de la doctrine du discernement. Cette nouvelle articulation, en superposant la dichotomie salut/damnation au problème de la distinction des esprits, fait monter les enjeux. À la question du discernement se lie maintenant celle du salut.

Ce n'est certainement pas un hasard si Origène, qui cite Hermas, semble soucieux de réaffirmer les prérogatives du libre arbitre (Marty 1958; Bertrand 2003a, 970-971) face à l'importance que prend l'influence des puissances spirituelles sur la destinée de l'âme (Sur les principes 3,2,4). Plus tard, Jean Cassien en arrivera à considérer cet exercitium cordis comme le propre du libre arbitre (Alciati 2009, 67). Insister sur l'impossibilité d'une connaissance pleine de la vérité - en l'espèce la nature réelle

9. Cela ressort de nombreux passages des premiers auteurs chrétiens, par exemple: Épître du Pseudo-Barnabé 18, Didaché 1,1-6,3; Doctrine des Apôtres 4-14, Constitution des Apôtres 1,1ss. 
d'une inspiration ou de l'esprit qui la transmet — risque de subordonner la capacité du choix moral qui découlerait de cette connaissance à une révélation supérieure: on se rapproche dangereusement de la gnose ${ }^{10}$, où justement le rôle du libre arbitre est plutôt effacé (Pétrement 1947, 3 ; Puech 1965). Et pourtant Origène maintient la pertinence du discernement dans la décision entre bien et mal (et donc au salut qui en résulte) sans délaisser la conception charismatique de cette capacité, ce qui la fait dépendre d'une initiative de la grâce. Il est donc impossible de discerner sans l'assistance de l'Esprit de Dieu (Homélies sur l'Exode 3,2). Ainsi, bien que formellement libre, l'arbitre humain risque, dans sa condition de déficience cognitive, de se retrouver sans défense face aux artifices des démons. Se profile ici, contre les puissances adverses, une guerre où triomphe non pas la volonté mais l'intelligence ${ }^{11}$. D'autre part, cette faculté de distinction - bien qu'attisée par la grâce - doit aussi être affinée par l'exercice, ce qui relève de l'agir humain. Telle est la conclusion qu'Origène tire de la lecture de l'Épître au Hébreux $(5,14)$, qu'il commente à plusieurs reprises (Commentaire sur saint Jean 6,267; Contre Celse 3, 53.60; 4,50; 6,13): "Mais la nourriture solide est pour les hommes faits, pour ceux dont le jugement est exercé par l'usage à discerner ce qui est bien et ce qui est mal

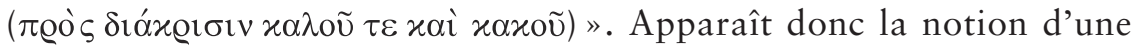
progression, un cheminement vers la perfection spirituelle qui est le fruit d'une purification (Monaci 2009, 16-18). Et, cette portion humaine du discernement se fonde sur une méthode tirée (Fr. 1Co 12,3-47: Jenkins 1907-1909) de la Première Épître de Jean (4,1-3: "Éprouver les esprits,

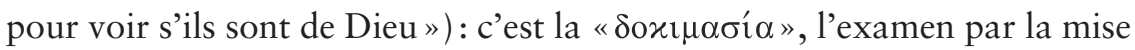
à l'épreuve (ce qui évoque sans doute le thème évangélique de l'observation a posteriori des fruits dérivant des inspirations). Il est intéressant de noter que même l'inspiration du prédicateur doit être éprouvée par ses auditeurs (Homélies sur Ézéchiel 2,2). C'est un détail essentiel, qui autorise une application élargie du discernement, où toute source d'influence exercée sur une personne doit être passée au crible par cette dernière, et ce, qu'il s'agisse des "esprits» ou des autorités religieuses. Il est aisé de saisir les raisons de l'oubli dans lequel tombera par la suite cette lecture du don spirituel, du moment qu'elle remet à chaque croyant — soit hors du mono-

10. Pour une comparaison entre clairvoyance gnostique et monastique, voir Painchaud et Wees (2002).

11. "Choisir librement entre le bien et le mal est, radicalement, un problème noétique " (Bertrand 2003a, 971). 
pole institutionnel - le soin de jauger ses «esprits » (ainsi que ceux de ses maîtres) et affirme par ce fait même la responsabilité individuelle du salut.

\section{La démonologie}

Dans le monachisme primitif (à partir du III-IV siècle) ${ }^{12}$, discerner les esprits c'est en premier lieu distinguer les bons des mauvais, et ce, afin d'éviter les pièges de ces derniers. La conception du diable comme d'un adversaire dissimulé et maquilleur que l'on a relevée d'abord chez Hermas puis dans la démonologie origénienne ${ }^{13}$ se trouve à être exaspérée dans la vision radicale qu'ont les premiers anachorètes de la vie ascétique. Cette dernière est représentée - à l'appui de plusieurs modèles bibliques ${ }^{14}$ comme une véritable guerre contre les démons, un combat secret ${ }^{15}$, une lutte sans quartier contre un ennemi caché. D'ailleurs, dans l'œuvre copte de Paphnutius (IV siècle), le qualificatif de «caché » (en copte: et thēp) est ajouté au nom de l'ennemi biblique (Amalek) ou de celui ethnique (les Nubiens) pour indiquer les démons qui agissent dans l'imposture (Vecoli 2003, 375). Du fait de sa capacité à se déguiser, le mal peut échapper au dépistage humain et induire en erreur même l'âme la mieux intentionnée: ce n'est que grâce à son discernement que le saint moine Macaire ( $\mathrm{IV}^{\mathrm{e}}$ siècle) reconnaît les trois démons qui lui rendent visite en tentant de se faire passer pour la Trinité (Vie de Macaire de Scété 20). En effet, la tradition monastique enseigne qu'il est impossible de se fier aux sens corporels pour repérer ce genre d'ennemis $\left(A\right.$. Alph. Élie $\left.3^{16}\right)$. Ce n'est que grâce au charisme divin que l'on est en mesure de percevoir la gravité de la menace: on racontait d'Antoine (251-357), l'anachorète considéré comme le père du

12. Pour une introduction générale au phénomène monastique des premiers siècles, voir Desprez (1998) et Filoramo (2010).

13. En ce qui concerne l'influence déterminante de l'œuvre d'Origène sur la pensée monastique, voir Rubenson (1990, 1999 et 2011).

14. Un exemple parmi légions, dans Paphnutius (2 [5r]), l'ancien moine enseigne au disciple les moyens de combattre les pensées des démons, ce qu'il nomme de «âpre combat ». Ce même combat est plus tard comparé à celui de Paul (dans Ep 6, 10-17) contre les dominations et les autorités $(4[9 \mathrm{v}-10 \mathrm{r}])$ et à celui de Moïse contre Amalek $(4[8 \mathrm{r}-8 \mathrm{v}])$.

15. Le Pseudo-Macaire (Ps-Mac. 21, 4) l'appelle « un autre combat, une autre lutte, une

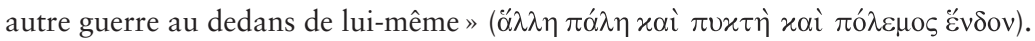

16. La rédaction des collections des apophtegmes se situe entre $\mathrm{V}^{\mathrm{e}}$ et $\mathrm{VI}^{\mathrm{e}}$ siècle, mais se nourrit d'une tradition orale précédente et qui fait référence à des personnages actifs à partir du IV siècle. Voir l'introduction de Guy au texte de la collection systématique: il y résume une vie de recherche sur la question. 
monachisme, qu'il était en mesure de voir les filets du malin s'étendre sur la terre entière $(A \text {. Alph. Antoine } 7)^{17}$. Dans ses Lettres, le saint insiste justement sur la nécessité d'en arriver, par la purification de l'ascèse, à une ouverture de l'« œil de l'âme»(Ep. Ant. 1,2: 5,5), ce qui constitue d'ailleurs un thème de dérivation évangélique (l'œil comme lampe du corps, dans Mt 6, 22-23: le lien est explicite chez Cassien, dans Conl. 2,2,51). D'autre part, Antoine insiste à de nombreuses reprises sur l'importance de connaître les espèces et les trames des anges du mal, car la connaissance démonologique apparaît comme le seul moyen d'échapper à leurs mensonges, autrement indétectables (Ep.Ant. 1,4; 4,6) ${ }^{19}$. Savoir humain ou révélation surnaturelle, le discernement est présenté comme l'instrument tactique par excellence (Ps-Mac. 11: IV-V ${ }^{\mathrm{e}} \mathrm{s}$.) dans le conflit qui oppose les chrétiens aux envoyés de Satan.

\section{Une technique}

Sur cette donnée de base de la doctrine ascétique des premiers moines, se greffent au moins deux développements essentiels. En premier lieu, la conception des démons est destinée à se diversifier selon les auteurs. Les puissances de l'air, véritables entités conscientes qui tourbillonnent entre ciel et terre (Daniélou 1956), finissent par être confondues avec le fruit de leurs chuchotements, c'est-à-dire les pensées impures (les " @u $\pi \alpha \varrho o i ̀ ~ \lambda o \gamma ı \sigma-$ roí » dans la V.A. 5,$4 ; 23,1$ ). La voix externe devient interne (à propos de Jean Cassien, sur ce point, voir Alciati 2009, 71)20. Se développe ainsi une sorte de psychologisation ${ }^{21}$ des "esprits" (Festugière 1962, 33 ss.; Brakke 2006) et la démonomachie devient une lutte contre les pulsions obscures

17. La capacité du saint à déceler la présence du mal là où les autres ne voient rien est un leitmotiv de la littérature hagiographique.

18. Avant lui, Macaire parlait d'une ouverture des "yeux du cœur" (Lettre à ses fils, 12).

19. Il a été dit que l'invisibilité des démons constituerait une spécificité des Lettres d'Antoine par rapport à la littérature monastique de l'époque. Je ne partage pas cette opinion, et de toute façon le problème n'est pas tant l'invisibilité mais l'habileté de camouflage et de déguisement (voir Bumazhnov 2007).

20. Dans les apophtegmes, il arrive que ces pensées impures soient considérées comme générées à l'intérieur du cœur de l'homme (A.Alph. Poemen 15).

21. Le terme est peut être un anachronisme conceptuel. Il est néanmoins intéressant de remarquer que - pour n'offrir que deux exemples - selon William James (1902, 68) la reconnaissance du surnaturel se fait par la constatation des sensations fortes qu'il provoque. Dans le même ordre de considérations, Rudolf Otto (1917) fonde son analyse du sacré sur la base des émotions qu'il provoque chez celui qui en fait l'expérience. 
qui malmènent l'intériorité humaine, "car aussi longtemps qu'un homme est retenu dans les choses visibles de ce monde, entouré des diverses chaînes de la terre, entraîné par les passions mauvaises, il ne sait même pas qu'il y a un autre combat, une autre lutte, une autre guerre au-dedans de luimême" (Ps-Mac. 21,4). Le combat est dans l'intellect (McMon. 139: $\mathrm{V}-\mathrm{VI}^{\mathrm{e}} \mathrm{s}$. $)^{22}$, et il se transforme en une œuvre de purification du soi qui est à la fois - fait paradoxal - condition nécessaire et aboutissement final du discernement (Vecoli 2006; 2007): il faut avoir le regard pur pour «voir ", mais peut être pur seul celui qui " voit ${ }^{23}$ ». Cette évolution est accompagnée par une interprétation technique du discernement qui remonte - nous l'avons vu - à Hermas. La mise en relation des inspirations surnaturelles avec les réactions émotives qu'elles déclenchent permet de forger une clé interprétative infaillible qui n'a plus rien de charismatique mais relève de la science expérimentale (encore que fondée dans le surnaturel): le calme est le signe de Dieu, alors que l'agitation relève du démon. Ainsi, dans les mêmes textes qui le présentent comme un dérivé de la grâce, le discernement s'affirme parallèlement comme une compétence qui dépend de l'expérience et se concrétise en une technique d'analyse du soi transmissible de maître à disciple ${ }^{24}$. Cette conception est vulgarisée en milieu chrétien par Athanase d'Alexandrie dans la Vie d'Antoine, et en milieu païen par le philosophe néo-platonicien Jamblique (vers 242-325) dans le traité les Mystères d'Égypte (2,3.10): pour s'y référer, les deux utilisent d'ailleurs le même terme ( $\delta \delta_{\imath} \alpha \dot{\gamma} v \omega \sigma \imath \varsigma »$, distinction $\left.{ }^{25}\right)$. On retrouve ce modèle binaire calme/agitation, entre autres ${ }^{26}$, chez le Pseudo-Macaire (Ps-Mac. 5,1.4; $6,3-4 ; 7,6)$, qui a le mérite d'expliciter $(P s-M a c .2,4)$ le lien exégétique avec l'image biblique de la brise légère dans laquelle Dieu se manifeste à Élie

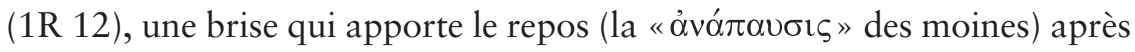

22. Ces mouvements impurs de l'âme sont classifiés et systématisés par Évagre le Pontique (346-399), notamment dans son écrit Des huit esprits de malice.

23. Cela est valable pour les paiens autant que pour les chrétiens, comme il est aisé de le constater en lisant Philostrate (II-III ${ }^{\mathrm{e}}$ s.): Apollonios de Tyane est dit avoir reçu de sa pureté la capacité de discerner dieux, héros, ou «fantômes immatériels qui simulent l'aspect humain" (Vie d'Apollonios de Tyane 6,11).

24. Une technique qui, dans sa dimension éthique, n'est pas sans nous évoquer l'examen de conscience de la philosophie classique (Hadot 1981).

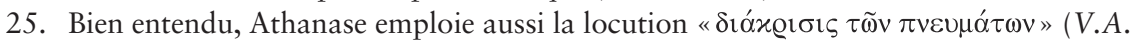
$22,3 ; 38,5 ; 44,1 ; 88,1)$.

26. Aussi, par exemple, la vie d'Évagre dans la version copte de la Histoire lausiaque (précédente au texte grec rédigé en 420) de Pallade d’Hélénopolis (p. 166-167); ou, plus tard, la Correspondance (VI ${ }^{\mathrm{e}}$ s.) de Barsanuphe et Jean de Gaza (124). 
la vaine turbulence de l'ouragan. Cet archétype est ensuite utilisé pour discerner les païens des chrétiens (Ps-Mac. 5,4), les premiers étant emportés dans la frénésie d'un mouvement sans fin alors que les vrais croyants vivent dans la paix d'une stabilité sans variation. À l'instar de la distinction des esprits de Paul, la reconnaissance de la véritable allégeance religieuse au IV-V $\mathrm{V}^{\mathrm{e}} \mathrm{s}$. n'est plus immédiate et s'opère désormais en observant certains signes ${ }^{27}$ : en effet, lors du triomphe post-constantinien du christianisme, nombreux sont ceux qui affichent à l'extérieur une foi en réalité vide et intéressée (Salamito 1995, p. 683). Malgré la finesse exégétique du PseudoMacaire, qui établit un pont théologique avec le récit d'Élie sur le mont Horeb, on ne peut nier que des motifs philosophiques grecs résonnent dans l'opposition chrétienne entre agitation démoniaque et tranquillité divine. En effet, l'équivalence agitation $>$ mouvement $>$ variation $>$ pluralités épuisement > mal opposée à celle calme> immobilité> stabilité> unité> repos> bien remonte, à travers le néoplatonisme, jusqu'aux syzygies pythagoriciennes. De toute évidence, dans une opposition ainsi structurée, il est aisé de classer le monothéisme chrétien du côté de l'unité et de reléguer le polythéisme païen du côté de la pluralité.

En tout état de cause, l'essence du discernement ne se laisse réduire à une représentation univoque. En somme, le don spirituel qui trouve son enracinement dans le charisme paulinien se structure simultanément comme une technique que l'on oserait presque définir de profane, si ce n'était que son fondement reste métaphysique. Cette technique prétend gérer un champ qui relève du spirituel, et doit pour cela se légitimer par la référence - que l'on pourrait qualifier d'abusive - à son antécédent scripturaire. D'un point de vue théologique, l'hésitation des textes entre charisme et technique manifeste l'impossibilité d'un choix qui obligerait à trancher entre grâce et libre arbitre, ce que les auteurs du monachisme ne font généralement pas $^{28}$. En tant qu'expression d'un savoir accumulé par l'expérience (ou appris d'un maître expérimenté), le discernement est une

27. Pallade d'Hélénopolis, dans le prologue à son Histoire Lausiaque (Pro 5-10), relayera une conception du discernement fondée sur la raison et l'interprétation des signes (en lien avec la physiognomonie). Voir aussi le «charisme» de saint Eutyme: "Il avait reçu de Dieu le charisme suivant: de l'aspect du corps visible il discernait les mouvements de l'âme et pouvait dire contre quelles pensées chacun devait lutter» (V.E. 29).

28. Voir, par exemple, la Lettre à ses fils $(4 ; 10)$ de Macaire: dans le parcours de perfectionnement spirituel du moine, les oscillations de la grâce — qui, selon les moments, vient en aide ou se retire pour laisser le solitaire lutter seul — exprime l'intention de l'auteur de conjuguer initiative divine et effort humain. Cette même dynamique se retrouve aussi dans les Lettres d'Ammonas (4,4.6). 
œuvre humaine qui s'apparente à la sagesse de la littérature sapientielle biblique, au sens où il se fonde sur une observation empirique qui permet d'éviter les dangers d'un mauvais choix (Crenshaw 2010, 11). En raison d'une certaine régularité phénoménique du monde, il suffit de savoir quels signes observer et comment les interpréter. Pour n'en mentionner qu'un, le comportement extérieur d'une personne est souvent révélateur de sa condition spirituelle: par exemple, une attitude querelleuse est clairement une manifestation du diable (McMon. 45). À ce même titre, l'ancien maître de Pachôme (v.292-346), Palamon ( $\left.G^{1} 8\right)$, est en mesure de dévoiler l'origine démoniaque d'un prodige opéré par un moine sur la base de son comportement arrogant ${ }^{29}$. Une méthode alternative assez récurrente consiste à interroger l'apparition ( $G^{1} 87$; V.A. 43,1), suivant en cela une ancienne

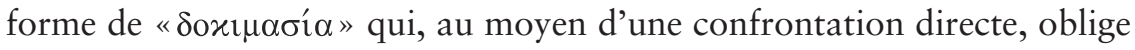
l'entité à révéler sa nature réelle ${ }^{30}$. Au-delà de sa forme ritualisée, cette procédure proche de l'exorcisme apprend au moine à focaliser une attention scrutatrice sur les pulsions néfastes qui ont tendance à se faufiler inaperçues dans son esprit: c'est la mise en pratique d'une attitude de vigilance extrême sur les pensées ${ }^{31}$.

Il y a un lien de cause à effet entre la pratique de l'ascèse et l'obtention d'un discernement ainsi conçu, puisque les efforts prolongés de la mortification et de la prière (sur cet aspect dans l'œuvre de Cassien: Levko 1996, 155-156) permettent l'acquisition d'une compréhension plus profonde de la vie spirituelle:

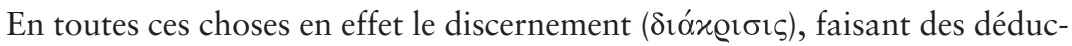
tions et des raisonnements, fait prospérer les bonnes choses et rend inefficaces les mauvaises. Il est impossible que le discernement vienne si tu ne fais

29. Le même critère, c'est-à-dire celui du comportement moral qui accompagne le prétendu charisme, était utilisé pour discerner la vraie prophétie aux origines du christianisme (notamment dans Didaché, 11).

30. Le passage biblique de Jos 5,3 ( Et il arriva, comme Josué était près de Jéricho, qu'il leva ses yeux et vit; et voici, un homme se tenait debout devant lui, son épée nue dans sa main; et Josué alla vers lui et lui dit: Es-tu pour nous, ou pour nos ennemis? ") est repris par Origène (Homélie sur les Nombres 27,11; Homélie sur Josué 6,2) et par Évagre (Lettre 11,3) pour exprimer la nécessité de discerner les esprits en les interrogeant.

31. Dans la lettre d'introduction $(11 ; 13)$ à son Histoire lausiaque (version copte), Pallade d'Hélénopolis explique que sans le discernement il est facile de créer en soi - sans s'en apercevoir - des mélanges impurs de pratiques saines et d'intentions contaminées, c'est-à-dire d'amalgamer élans bons et mauvais. Le discernement surveille et met à nu les intentions du soi. 
pas tout ce qui l'accompagne [(sa liturgie, $\lambda \varepsilon \imath \tau o v \varrho \gamma i ́ \alpha)]$ : d'abord [rechercher] la solitude; la solitude engendre l'ascétisme et les larmes; les larmes engendrent la crainte; la crainte engendre l'humilité et la prévision ( $\pi$ @oo@ãv); la prévision engendre la charité; la charité rend l'âme saine et impassible; alors l'homme comprend qu'après toutes ces choses, il n'est pas loin de Dieu ». (Ammonas, Sur l'allégresse de l'esprit de celui qui commence à servir Dieu 60: 2e moitié du IV ${ }^{\mathrm{e}}$ siècle)

Le monachisme opère une "révolution symbolique » : le discernement n'est plus le signe d'une vocation (Paul) mais le produit d'un parcours de purification, il n'est plus le gardien de l'harmonie communautaire (Paul) mais l'instrument - obtenu par la sueur et les larmes — d'un salut individuel. Nous ne pouvons qu'avancer des hypothèses quant aux raisons de ce changement de conception.

L'émergence d'une interprétation technique du discernement semble répondre à des nécessités concrètes apparues lors du développement du monachisme. L'ascèse du solitaire est une œuvre exigeante, voire éreintante (le « $\pi$ óvos» monastique), pratiquée dans le cadre d'un genre de vie qui put apparaître extrême à ceux qui en furent les témoins ${ }^{32}$. Une surveillance étroite et continue s'avère nécessaire. Dans ce milieu anachorétique à l'origine non rigidement structuré (Gould 1993, Caner 2002), on apprend à

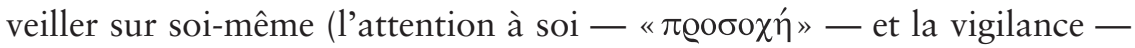

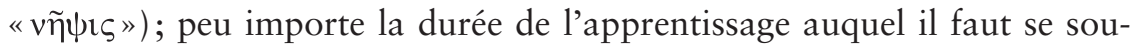
mettre, on finit tôt ou tard par devoir assumer la responsabilité de sa propre destinée. Or, les mortifications parfois extrêmes de ce genre de vie font prendre conscience aux théoriciens qu'il y a une dimension psychologique du salut que l'on ne peut négliger: la perfection spirituelle doit aller de pair avec le bienêtre mental. Le discernement, qui par ailleurs sert aussi à dépister les vraies maladies des imitations illusoires ( $G^{1} 52-53$; Bars. Corr. 42, 78, 153), se dresse comme une sentinelle contre les appâts de la folie ou du désespoir, qui risquent de suborner les athlètes de l'esprit (sur les maladies spirituelles, voir Larchet 1997). Il faut bénéficier d'un savoir concret dont l'efficacité ne peut dépendre des caprices de la grâce. Et la transmission de ce savoir motive justement la rédaction des Lettres d'Antoine. L'enjeu est l'acquisition d'une compétence qui sous cet aspect

32. Malgré la tendance déconstructionniste d'une part de la littérature anglo-saxonne à l'égard de la vie monastique (Goehring 1993), l'affirmation (contre toute herméneutique radicale) d'un lien — si tenu soit-il — entre discours et réalité oblige à prendre au sérieux cette perception qui transparait dans les sources (efficacement relatée, pour ce qui est des Pères du désert, dans Regnault 1990). 
demeure apolitique, car elle n'alimente aucune autorité contraignante sur les autres. Ce n'est d'ailleurs pas une coïncidence si la sphère d'application de cet outil de l'ascèse reste, à ce stade, exclusivement personnelle: dans une certaine perspective sociologique, on pourrait parler à ce propos d'une phase d' "autoconsommation" religieuse (Bourdieu 1971, 305). Tout au plus (mais nous touchons ici ses limites extrêmes ${ }^{33}$ ), l'expérience d'une longue pratique peut permettre au maître d'appliquer cette science sur un nombre restreint de disciples, avec qui il entretient une relation personnelle de direction spirituelle (selon la définition plutôt resserrée de Hausherr 1955, 10: "Nous réservons [ce nom] aux relations entre un maître instruit et expérimenté dans les voies de l'esprit et un disciple désireux de profiter de cette science et de cette expérience»). L'interprétation technique du discernement résulte donc d'un développement lié à l'histoire de l'ascétisme. Plus spécifiquement, elle s'affirme suite à la découverte monastique de la complexité et de l'opacité d'un univers intérieur humain qui se révèle exceptionnellement résistant à la parfaite unification à Dieu (ce que les

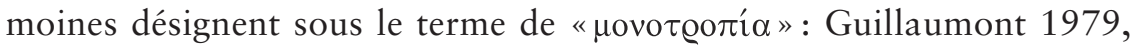
55-58).

\section{L'économie de l'invisible}

La théorie monastique qui se dégage du fondement démonologique susmentionné implique un développement ultérieur, dans une certaine mesure contradictoire par rapport à celui que nous venons de souligner. En effet, ce deuxième développement se situe à l'opposé de la réduction psychologisante des démons ou de l'interprétation de signes phénoméniques. La guerre cachée qui rend le discernement indispensable relaye une Weltanschauung très répandue dans l'antiquité tardive: c'est ce que l'on pourrait appeler une "économie de l'invisible». Contrecoup d'un recul du rationalisme grec face à la diffusion des nouveaux "cultes orientaux » (dont le christianisme: McMullen 2011, 132-133) ou répercussion de l'entrée dans un "âge d'angoisse" provoquée par la crise de l'empire (Dodds 1965), dans tous les cas (voir aussi Marrou 1977) nos sources montrent clairement une accentuation de la place occupée par l'invisible

33. D'ailleurs, les apophtegmes ne s'embarrassent pas de mettre en exergue un maître sans discernement qui s'avère incapable de percevoir la nature divine des visions de son propre disciple (A. Alph. Zacharie 4). Nous sommes loin de la "cardiognostie" surnaturelle qui s'affirme dans le monde cénobitique pachômien (voir Amm. 2-3 ou Bo 107, cité plus en avant). 
dans la mécanique du réel: "Renonce au visible, afin de voir l'invisible; qu'il y ait une garde en ton cœur, afin que tu connaisses ceux qui te troublent; car au parfaits revient la nourriture solide, eux qui par habitude ont entraîné leurs sens à discerner ( $\delta$ róx la garde du cœur» (Étienne de Thèbes, Discours ascétique 1-3: v-vi ${ }^{\mathrm{e}} \mathrm{s}$.). Pour la mentalité ambiante, les engrenages essentiels de l'univers se situent dans une dimension éthérée, inaccessible à ceux qui ne possèdent pas une deuxième vue. À partir des grandes élaborations cosmologiques jusqu'à l'anatomie du corps humain, le matériel est sous l'emprise du spirituel. Des forces invisibles exercent une influence déterminante sur le fonctionnement du monde. Le déterminisme astral infuse les différentes traditions religieuses de l'époque, comme on peut le constater dans le domaine de la mélothésie: la tradition juive (Sefer Yetsirah 49), les textes hermétiques (Corpus Hermeticum 13; 16), les spéculations manichéennes (Kephalaia 70) et la doctrine chrétienne (Origène, Contra Celsum 8, 58), tous s'accordent sur le fait que les membres du corps humain sont habités par des démons qui en influencent l'action de façon contraignante autant qu'ils gouvernent le mouvement des astres. La relation étroite entre microcosme et macrocosme crée un effet de résonnance: s'il est au départ perçu de

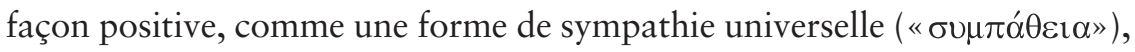
il finit néanmoins par être compris comme l'expression d'un déterminisme

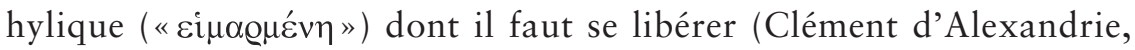
Excerpta ex Theodoto 25,2). L'effort ascétique des moines peut donc être compris (Ep. Ant. 1,4; Ps-Athanase, Vie de Synclétique 81-87: ve s.) comme une œuvre de libération de l'être humain de l'emprise qu'exercent sur lui les puissances inférieures. Ceci vaut pour le monde aussi bien que pour l'individu. Une grave épidémie dans la ville d'Éphèse, aux yeux éclairés du philosophe néopythagoricien Apollonios de Tyane, se révèle comme étant provoquée par un démon qui a pris la forme d'un vieillard (Philostrate, Vie d'Apollonios de Tyane 4,10). À Constantinople, seul le saint chrétien comprend que l'origine d'un grand incendie qui fait des ravages en ville est reliée à la gravité des péchés du peuple $(M r A c 31$ : v.400-v.484). La structure binaire du réel, divisée entre le visible — pourtant illusoire - et l'invisible — mais vrai —, s'intègre parfaitement à la théologie chrétienne, qui en fait d'ailleurs le soubassement de la doctrine eucharistique de la transsubstantiation: au saint moine qui est capable de discerner, les espèces du pain et du vin apparaissent pour ce qu'elles sont réellement (le corps et le sang du Christ) dans toute leur répugnante matérialité (A. Alph. Daniel 7). Cette question reviendra par la suite, lors de la 
controverse sur l'eucharistie soulevée par les thèses de Bérenger de Tours $\left(\mathrm{XI}^{\mathrm{e}} \mathrm{s}\right.$.) : l'économie de l'invisible triomphera une fois de plus.

Le chiasme qui s'établit entre visible/invisible et faux/vrai reflète le sentiment de désorientation ressenti dans un monde qui change. Le pluralisme religieux ainsi que le fractionnement des chrétiens fragilise l'idée d'une vérité exclusive autoévidente: peut-être est-il possible de dire, mutatis mutandis, que les «structures de plausibilité » (Berger 1967) de la vérité chrétienne se trouvent paradoxalement et temporairement effritées sous l'effet de la transition conflictuelle vers un empire christianisé. Un obscur moine copte l'écrit en s'adressant à son disciple: "Ne te laisse pas prendre par la confusion qui existe» (Paul de Tamma, De Cella 30: Iv e s.). Un auteur lié au monde pachômien $\left(2^{\mathrm{e}}\right.$ moitié du $\mathrm{IV}^{\mathrm{e}} \mathrm{s}$.) le rapporte de façon particulièrement efficace quand il décrit une vision de Pachôme, survenue au moment où celui-ci, confus, doit décider à quelle doctrine adhérer: le monde est plongé dans les ténèbres et à des endroits différents s'élèvent des

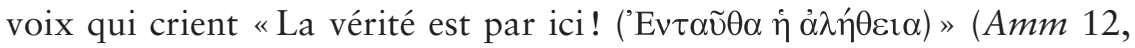
18-22). Sans l'aide d'un surplus de connaissance, il est impossible de s'orienter. Et pourtant, à l'œil purifié du maître les enjeux de l'aventure humaine se montrent de façon très nette. Ainsi, le père Moïse (A. Alph. Moïse) peut révéler à son jeune disciple que le désert des moines n'est pas vide, encore moins neutre: les armées des anges et des démons se font face aux deux horizons, prêtes à engager le combat sur les âmes des hommes (voir aussi: Ps-Mac. 14,4). À la confusion humaine, le discernement surnaturel oppose l'alternative très claire d'un affrontement entre lumière et ténèbres. La technique est ici doublée d'une dimension charismatique. L'enjeu est d'abord épistémologique, du moment que l'affirmation d'une connaissance ultime accessible par la seule entremise d'une révélation situe l'homme dans une condition d'insuffisance cognitive fondamentale face à l'établissement de la vérité ${ }^{34}$ : le discernement comme charisme dissimule une déficience humaine.

\section{La décision}

Le problème en amont, celui que la théologie monastique doit résoudre, consiste en la difficulté à ramener un réel que l'on découvre de plus en plus

34. D'aucuns expliquent d'ailleurs l'affirmation d'une connaissance révélée dans les milieux esséniens par l'échec de l'optimisme épistémologique de la littérature sapientielle biblique (von Rad 1971). 
complexe et impénétrable à la simplicité claire d'une dichotomie binaire rigide: l'ambiguité du monde gagne. Et pourtant, il faut pouvoir établir une différence (Waaijman 1997, 6) là où on ne voit qu'indifférence, sous peine de perdre toute capacité d'orientation dans ce cadre de référence dominé par l'alternative salut/damnation. L'exaspération monothéiste du monachisme ne peut accepter - sauf exceptions - l'existence de ce que

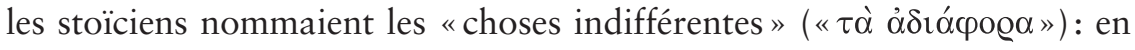
toute situation, même la plus insignifiante, il doit être possible de déterminer entre les innombrables voies parcourables l'unique droit chemin, celui que Jean Cassien appelle la "via regia» (Conl. 2,2,4, en se référant à Nm $\left.20,17^{35}\right)$. On présuppose de fait l'existence d'une mesure précise de la perfection parmi toutes les tendances et forces en action. À l'origine, il s'agit de la très délicate question de l'équilibre (entre rigorisme et laxisme, pour ainsi dire) dans le régime ascétique. La quête d'un juste milieu adapté à chacun des anachorètes du désert inspire à Cassien la comparaison des moines avec des funambules (Conl. 23,9,1). Mais de là, la fonction de trouver la mensura parfaite s'élargit à toute problématique humaine. Et quand la dichotomie entre vérité et erreurs s'avère laborieuse, la différence est transférée à un degré de subtilité plus élevé. C'est ce que fait Isaïe de Scété (Asceticon 2,7) lorsqu'il oppose nature et contre-nature à l'égard d'un même objet en soi impossible à classer, c'est-à-dire le sentiment de la colère. En tant que réaction émotive humaine, la colère maintient son ambivalence jusqu'au moment où sont établies les conditions de son exercice: le clivage intervient alors entre une colère naturelle qui s'embrase contre le péché, et une colère contre-nature qui surgit des frustrations personnelles.

À chaque pas, l'homme est appelé à faire son choix entre bien et mal face à un réel en perte de transparence. Les données empiriques paraissent de moins en moins pertinentes, et par conséquent la raison dialectique aussi. Antoine refuse d'ailleurs de s'aventurer sur ce terrain — c'est-à-dire la confrontation dialectique - avec les philosophes païens qui lui rendent visite: c'est sa puissance thaumaturgique, non pas son raisonnement, qui les réduira au silence (V.A. 72-80). Le discernement finit par renvoyer à une dimension métaphysique la décision impossible à prendre sur la base du seul contexte profane: "En cas de crisis, la diacrisis est nécessaire. La crise est ambigüe par nature, il faut savoir la discerner, puis l'assumer: elle devient alors étape de progrès» (Miquel 1997, 105).

35. Alciati $(2009,73)$ souligne le lien avec la doctrine aristotélicienne de la «via media». 
La diakrisis fait donc progresser, ce qui - hors d'une perspective téléologique - signifie avancer. Et avancer implique nécessairement décider. Le discernement est donc lié à la décision. Il sert la décision, il la rend possible là où ses bases sont de fait insuffisantes: voir la bonne voie entre plusieurs, réduire le choix entre une seule bonne (la meilleure) et toutes les autres mauvaises (ou simplement moins bonnes), cela sert à procéder audelà des intersections de la vie quand celles-ci présentent des ambiguïtés insolubles. On peut remettre cette décision dans les mains de l'homme, en élaborant une technique qui se construit sur la base de l'expérience et se transmet par l'enseignement, ou la faire dépendre d'une volonté divine qui se manifeste à travers le charisme spirituel. Sans la conviction qu'il existe une seule décision juste à prendre en toute chose, la conscience de la complexité et du relativisme du réel risque de provoquer une irrésistible paralysie chez celui qui avance. En ce sens, le discernement incarne une fonction centrale (et trop souvent sous-estimée) de la religion, déjà repérée par l'anthropologie fonctionnaliste (Malinowski 1925, 82-83): libérer l'homme de la paralysie face à l'impondérable ${ }^{36}$. Lorsque la connaissance «scientifique» permettant de diriger l'action humaine fait défaut, la magie (que Malinowski sépare encore de la religion) prend la relève en maintenant l'être humain en mouvement, soit en mode positif d'expérimentation; et ce, afin de contrer le sentiment d'impuissance ou la peur qui bloquerait toute initiative. L'ignorance profane ouvre la porte à la connaissance sacrée: c'est un principe ancien, que l'on retrouve à l'œuvre dans toute pratique divinatoire ${ }^{37}$.

L'essor du discernement spirituel dans la tradition monastique primitive est lié à la conscience de l'ambiguïté irréductible du réel. Si l'on maintient la pertinence de l'alternative rigide bon/mauvais face à toute situation complexe, s'impose obligatoirement une solution univoque qui ne saurait être trouvée sans la conviction de son existence (elle doit exister!) et de son accessibilité (il faut pouvoir la connaître). Le choix appelle une décision et

36. Le fait que nous ne pouvons fonctionner (sans être paralysés) sans un bagage d'idée abstraites - donc invisibles - qui confère un sens aux objets concrets de notre expérience, ceci est affirmé - sur un plan psychologique - par William James, qui nomme «helplessness» (James 1999, 65) le danger dans lequel on encoure sans ce bagage mental. Ici James veut prouver que malgré les affirmations de Kant sur l'inconsistance du religieux celui-ci au contraire a un impact très concret dans la vie de l'être humain.

37. Un principe anthropologique? Les sciences cognitives ont insisté sur la tendance humaine à postuler l'existence d'entités inobservables pour expliquer des phénomènes observables (Horton 1967, repris dans Guthrie 1993). 
la décision ne peut s'imposer que si elle jouit d'une autorité contraignante qui légitime le décideur (face à lui-même ou face aux autres). La question est de savoir sur quoi repose cette autorité.

De fait, le discernement permet de décider dans ce que Carl Schmitt appelait la «situation exceptionnelle» (Schmitt 1988, 15). Le philosophe juriste allemand avait souligné, en rapport à la figure du souverain, l'essence de son autorité dans le cas d'exception: ici, la décision du souverain ne se fonde sur rien d'autre que la décision elle-même; car en effet, si elle devait émaner de conditions préalables attendues, elle ne serait alors que l'application d'un protocole. Ainsi, quand la situation n'indique pas clairement une action directement conséquente (qui ne serait autre qu'une réaction), s'ouvre une crisis qui attire le spectre de l'immobilité (corollaire de l'impossibilité à justifier toute décision). Il n'y a alors que deux alternatives: soit on accepte le caractère totalement autoréférentiel de la décision (qui est celle du souverain de Schmitt) au nom d'une autorité préalablement attribuée au décideur, soit celui-ci est désigné par la reconnaissance d'un accès (fruit d'une technique ou d'un charisme) à un savoir d'origine surnaturelle. La légitimation de ceux qui entourent le décideur est toujours nécessaire. Dans le cas d'une fondation de l'autorité par le discernement spirituel, il faut que celui-ci soit accepté comme légitime et efficace ${ }^{38}$. Or, pour que soit admise la capacité à voir et indiquer le bon chemin là où la "science» fait défaut, doit avoir lieu — si l'on se situe à l'extérieur d'une perspective croyante - une "méconnaissance des limites de la connaissance ». Phénomène de mystification s'il en est, c'est bien cette méconnaissance qui rend "objective» (ce qui signifie viable à des fins de décision) une vérité qui ne l'est pas (Bourdieu 1971, 310).

En revenant à Schmitt, le texte allemand de sa Théologie politique souligne (voir la note du traducteur français dans Schmitt 1988, 15) que non seulement il y a une décision dans la situation exceptionnelle, mais qu'il y a une décision à propos de la situation exceptionnelle, c'est-à-dire que le souverain décide quand la situation est exceptionnelle avant même de décider comment la résoudre. Par suite, non seulement toute situation où l'on manque de points de repères immédiatement évidents est potentiellement exceptionnelle mais l'est également celle qui est déclarée telle par celui qui détient l'autorité.

38. Un problème dont étaient conscients les moines du IV ${ }^{\mathrm{e}} \mathrm{s}$ : : «J'ai demandé à Ausonios si un homme peut réellement voir les choses cachées dans les cœurs des hommes et de me convaincre pleinement sur la base des Écritures» (Amm. 16). 
Dans le cas du discernement, la situation exceptionnelle est à l'origine déterminée par la manifestation des "esprits ", qui représentent effectivement une exception par rapport au fonctionnement des facultés normales de l'être humain. Or, dans une perspective religieuse où s'affirme l'économie de l'invisible, c'est-à-dire où les données empiriques — considérées illusoires — n'entretiennent plus un rapport de causalité nécessaire avec les fondements du réel (à tout le moins de ce réel qui compte), toute situation finit par tomber dans la catégorie de l'exception - une sorte d'occasionnalisme sui generis - et entrer ainsi dans la sphère d'application du discernement spirituel. Si l'on ajoute à cela la dimension fortement charismatique - cette fois au sens wébérien du terme - du monachisme primitif (Vecoli 2006), c'est-à-dire son organisation originairement souple et axée sur le prestige personnel de certains personnages extraordinaires, il est aisé de saisir comment peut avoir lieu en ce contexte une hypertrophie du discernement, le charisme de l'exception. Ce don spirituel finit par exprimer la supériorité d'une connaissance divine qui ne s'applique plus seulement aux cas ambigus, mais - par un effet de débordement - provoque la mise en question de toute forme de connaissance empirique. De ce développement du discernement s'ensuit l'affirmation d'une autorité exceptionnelle et incontestable, qui investit la gestion de la communauté monastique entière et la société en général. Quand Pachôme reproche au moine à qui il a délégué d'examiner les postulants à la vie monastique de laisser entrer la mauvaise herbe dans la congrégation, celui-ci s'exclame, exaspéré: "comme si j'avais moi aussi le charisme que le Seigneur t'a accordé de reconnaître les bons des mauvais!» (Bo 107). L'épisode relaté par Cyrille de Scythopolis (V.E. 44), où le saint supérieur du monastère reproche à ses moines d'être partis chercher de l'eau lors d'une sècheresse - car lui sait qu'il suffit de prier pour faire pleuvoir -, montre toute l'ampleur de la question. Très tôt, cette prodigieuse clairvoyance trouvera des applications politiques, et le saint deviendra le conseiller des commandants militaires ou des gouverneurs provinciaux (c'est le cas de Jean de Lycopolis, dans Hist. Mon. 1,1.2; ou de Chénouté dans V.S. 106-108).

Dans la reconnaissance que la société accorde à son charisme spirituel, le saint moine de l'antiquité tardive trouve un réservoir inépuisable d'influence sociale, voire même politique, qui le caractérise comme le troisième centre de pouvoir, après ceux de l'administration impériale et de l'institution ecclésiastique (sur les fonctions sociales et civiles du saint de l'antiquité tardive: Brown 1971 et 1987). Par la suite, l'institutionnalisation du monachisme amènera l'activation de stratégies d'endiguement ou de 
détournement de ce que l'on percevra comme les excès incontrôlables de l'omnipuissance du discernement. Ce charisme devient rapidement un bien de salut et structure par ce fait même un champ religieux: s'ensuit la lutte pour la monopolisation de sa gestion. Mais c'est là toute une autre histoire.

\section{Sources (et abréviations)}

AMmon, Lettre $(A m m)$ :

Goenring, J. E. (1986), The Letter of Ammon and Pachomian Monasticism, Berlin/New York, De Gruyter.

Ammonas, Lettres (Ams):

Nau, F. (1916), "Ammonas successeur de Saint Antoine. Textes grecs et syriaques ", Patrologia Orientalis, 11/4, p. 391-487 [Texte grec et syriaque et traduction française].

Antoine, Lettres (Ep.Ant.):

GaRitTe, G. (1955), Lettres de saint Antoine. Version géorgienne et fragments coptes, Louvain, Peeters (CSCO 148-149) [Texte grec et traduction française].

APOPHTEGMES DES PÈRES :

Série Alphabétique (A. Alph.): Migne, J. P., Patrologia Graeca LXV [Texte grec]; GuY, J.-Cl. (2000), Paroles des anciens. Apophtegmes des pères $d u$ désert, Paris, Seuil [Traduction française].

Série Systématique (A. Syst.): Guy, J.-Cl. (1993-2005), Les Apophtegmes des pères, Paris, Cerf [Texte grec et traduction française].

Athanase D'Alexandrie, Vie d'Antoine (V.A.):

Bartelink, G. J. M. (1994), Athanase d'Alexandrie. Vie d'Antoine, Paris, Cerf [Texte grec et traduction française].

Barsanuphe et JeAn de gaza, Correspondance (Bars.Corr.):

Neyt, F., De Angelis-Noah, P. et Regnault, L. (1997-2002), Barsanuphe et Jean de Gaza. Correspondance, Paris, Cerf [Texte grec et traduction française].

Cassien, Jean, Conférences (Conl.):

Petschenig, M. et Pichery, E. (1955-1971), Jean Cassien. Conférences, Paris, Cerf.

Cyrille de Scythopolis, Vie d'Eutyme (V.E.):

Festugière, A.-J. (1962), Les moines d'Orient. III. Les moines de Palestine $1^{\text {er }}$ fascicule, Cyrille de Scythopolis: Vie de saint Euthyme, Paris, Cerf [Traduction française].

Schwartz, E. (1939), Kyrillos von Skythopolis, Leipzig, J. C. Hinrichs [Texte grec]. 
ÉTIENNE DE ThÈBes, Discours ascétique:

Des Places, É. (1969), "Le Discours ascétique d'Étienne de Thèbes. Texte grec inédit et traduction ", Le Muséon 82, p. 35-59.

Histoire des moines en ÉGYPTE (Hist.Mon.):

Festugière, A-J. (1971), Historia Monachorum in Aegypto, Bruxelles,

Société des Bollandistes [Texte grec et traduction française].

ISAÏE DE SCÉTÉ, Recueil ascétique:

Regnault, L. et De Broc, H. (1976²), Abbé Isaïe. Recueil ascétique, Bégrolle-en-Mauges, Abbaye de Bellefontaine [Traduction française].

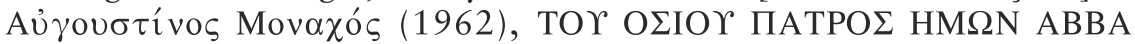

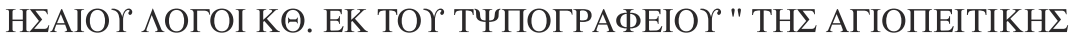

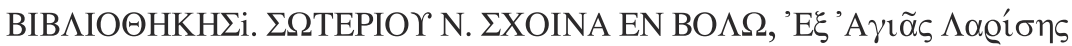
$\left(\Theta \varepsilon \sigma \sigma \alpha \lambda i^{\prime} \alpha \varsigma\right)$.

Macaire de SCÉTÉ, Lettre à ses fils:

Wilmart, A. (1920), "La lettre spirituelle de l'abbé Macaire», Revue d'ascétique et mystique, 1, p.58-83 [Texte latin].

Louf, A. (1985), "Saint Macaire l'Égyptien. Lettre à ses fils », dans. AA.VV., Les lettres des pères du désert, Bégrolles-en-Mauges, Abbaye de Bellefontaine, p.61-81 [Traduction française].

Macaire Pseudo-, Homélie spirituelles (Ps-Mac).

Deseille, P. (1984), Les homélies spirituelles de saint Macaire, Bégrolles-enMauges, Abbaye de Bellefontaine [Traduction française].

Migne, J.-P. (1903), Patrologia Graeca XXXIV, Paris, Garnier, col. 449822 [Texte grec].

Mani, biographie dans le Codex Manichaicus Coloniensis (CMC)

Gnoli, F. (2003), dir., Il Manicheismo - Vol. 1 - Mani e il manicheismo, Milano, Fondazione L. Valla, Mondadori [Texte grec et traduction italienne].

MARC le MoINe, La loi spirituelle (McMon):

Durand, G. M. de (1999), Marc le moine. Traités I, Paris, Cerf [Texte grec et traduction française].

PACHôme, Écrits:

Lefort, L. T. (1956), Euvres de S. Pachôme et de ses disciples, Louvain, Peeters [Texte copte et traduction française].

Pallade d’Hélénopolis, Histoire Lausiaque (Hist.Laus.):

Mohrmann, C. et Bartelink, G. J. M. (1974), Palladio. La storia lausiaca, Milano, Mondadori [Texte grec et traduction italienne].

Molinier, N. (1999), Pallade d'Hélénopolis. Histoire Lausiaque, Bégrollesen-Mauges, Abbaye de Bellefontaine [Traduction française du texte grec]. 
Bunge, G. et De Vogüé, A. (1994), Quatre ermites égyptiens. D’après les fragments coptes de l'Histoire Lausiaque, Bégrolles-en-Mauges Abbaye de Bellefontaine [Traduction française du texte copte].

Paphnutius, Histoires des moines de la Haute Égypte:

Wallis Budge, E. A. (1977), Coptic Texts. Volume V. Miscellaneous Texts in the Dialect of Upper Egypt, New York, AMS Press, p. 432-495 [Texte copte et traduction anglaise].

Paul de Tamma, Oeuvres:

Orlandi, T. (1988), Paolo di Tamma. Opere, Roma, C.I.M. [Texte copte et traduction italienne].

Vie de Chenouté (V.S.):

LEIPOLDT, J. (1906), Sinuthii archimandritae vita et opera omnia. I. Sinuthii Vita boahirice, Paris, E Typographeo Reipublicae [Texte copte].

Orlandi, T. (1984), Vite di monaci copti, Roma, Città Nuova Editrice, p. 127-184 [Traduction italienne].

Vie de Marcel l'Acémète $(M r A c)$ :

Dagron, G. (1968), «La Vie ancienne de saint Marcel l'Acémète », Analecta Bollandiana, 86, p. 287-321 [Texte grec].

Baguenard, J. M. (1990), Vies des saints Alexandre, Marcel et Jean Calybite, Bégrolles-en-Mauges, Abbaye de Bellefontaine [Traduction française].

Vies de PACHÔME

Première Vie grecQue $\left(G^{1}\right)$ :

Halkin, F. (1932), Sancti Pachomii Vitae Graecae, Bruxelles, Société des Bollandistes [Texte grec].

Festugière, A.-J. (1962-1963), Les moines d'Orient IV/2. La première vie grecque de saint Pachôme, Paris, Cerf [Traduction française].

VIE COPTE BOHAÏRIQUE $(B O)$ :

LEForT, L. T. (1966), Les vies coptes de saint Pachôme et de ses premiers successeurs, Louvain, Bureau du Muséon [traduction française des textes coptes].

\section{Références}

Alciati, R. (2009), "Il de discretione di Cassiano e la sua influenza", Rivista di storia del cristianesimo, 6, p. 65-98.

Attridge, H. W. (2000), "Didache", dans L. H. Schiffman et J. C. Vander Kam, Encyclopedia of the Dead Sea Scrolls, Oxford, Oxford University Press, p. 204-205. 
Berger, K. (1998), "Carisma », dans H. Balz et G. Schneider, dir., Dizionario esegetico del Nuovo Testamento, Vol. 2, Brescia, p. 18861889.

Berger, P. (1967), The Sacred Canopy. Elements of a Sociological Theory of Religion, Garden City, Doubleday.

Bertrand, D. (2003a), "Origène et le discernement des esprits ", dans L. Perrone, dir., Origeniana Octava, Leuven, Leuven University Press, p. $969-975$.

(2003b), «Le discernement des esprits et la portée théologique de la Vie d'Antoine par Athanase ", dans P. LARDET, dir., La tradition vive. Mélanges d'histoire des textes en l'honneur de Louis Holtz, Turnhout, Brepols.

Bourdieu, P. (1971), "Genèse et structure du champ religieux ", Revue française de Sociologie, 12, p. 295-334.

Brakke, D. (2006), Demons and the Making of the Monk. Spiritual Combat in Early Christianity, Harvard University Press, Cambridge/London.

Braudel, F. (1949), La Méditerranée et le monde méditerranéen à l'époque de Philippe II, Paris, Armand Colin.

Brown, P. (1971), "The Rise and Function of the Holy Man in Late Antiquity ", Journal of Roman Studies, 61, p. 80-101.

(1987), "The Saint as Exemplar in Late Antiquity», dans J. S. Hawley, dir., Saints and Virtues, Berkeley, University of California Press, p. 3-14.

Bumazhnov, D. (2007), "The Evil Angels in the "Vita" and the "Letters" of St. Antony the Great. Some Observations concerning the Problem of the Authenticity of the "Letters" ", Zeitschrift für antikes Christentum, 11 , p. 500-516.

Caner, D. (2002), Wandering, Begging Monks. Spiritual Authority and the Promotion of Monasticism in Late Antiquity, Berkeley/Los Angeles/ London, University of California Press.

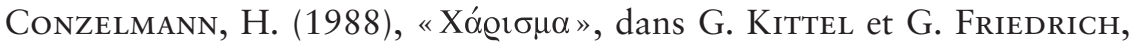
dir., Grande Lessico del Nuovo Testamento, Vol. 15, Brescia, Paideia, p. 606-616.

Crenshaw, J. L. (20103), Old Testament Wisdom. An Introduction, Louisville, WJK Press.

Daniélou, J. (1956), "Les démons de l'air dans la 'Vie d'Antoine' ", dans B. Steidle, dir., Antonius Magnus Eremita, Roma, Herder (Studia Anselmiana), p. 135-145. 
De Certeau, M. (1995 [1982]), La Fable mystique. XVI et XVII siècles, Paris, Gallimard.

Desprez, V. (1998), Le monachisme primitif. Des origines jusqu'au concile d'Éphèse, Bégrolles-en-Mauges, Abbaye de Bellefontaine.

Dodds, E. R. (1965), Pagans and Christians in an Age of Anxiety. Some aspects of religious experience from Marcus Aurelius to Constantine, Cambridge, Cambridge University Press.

Dörries, H., et al. (1981), "Gnade", dans T. Klauser et al., dir., Reallexicon für Antike und Christentum, Vol. 11, Stuttgart, 1981, p. 313-446.

Ducros, X. (1953), "Charismes", dans M. Viller et al., dir., Dictionnaire de spiritualité ascétique et mystique. Doctrine et histoire. II, Paris, Beauchesne, col. 503-507.

Festugière, A.-J. (1962), "Le moine et les démons ", Les moines d'Orient, I. Culture ou sainteté. Introduction au monachisme oriental, Paris, Cerf, p. 23-39.

Filoramo, G. (2010), Monachesimo orientale. Un'introduzione, Brescia, Morcelliana.

Goenring, J. E. (1993), "The Encroaching Desert. Literary Production and Ascetic Space in Early Christian Egypt ", Journal of Early Christian Studies, 1, p. 281-296.

Giot, J. et Sснотте, J.-C. (1999), Langage, clinique, épistémologie. Achever le programme saussurien, Bruxelles, De Boeck.

Gould, G. (1993), The Desert Fathers on Monastic Community, Oxford, Clarendon Press.

Guilhaumou, J. (2006), Discours et événement. L'histoire langagière des concepts, Besançon, Presses Universitaires de Franche-Comté.

Guillaumont, A. (1979), "Monachisme et éthique judéo-chrétienne », dans Aux origines du monachisme chrétien. Pour une phénoménologie du monachisme, Bégrolles-en-Mauges, Abbaye de Bellefontaine, p. 47-66.

Guillet, J. (1957), «Discernement des esprits. I. Dans l'Écriture», dans M. VILler et al., dir., Dictionnaire de spiritualité ascétique et mystique. Doctrine et histoire. III, Paris, Beauchesne, col. 1222-1247.

Guthrie, S. (1993), Faces in the Clouds. A New Theory of Religion, New York, Oxford University Press.

Надот, Р. (1981), «Exercices spirituels antiques et philosophie chrétienne", Exercices spirituels et philosophie antique, Paris, Études Augustiniennes, p. 59-74. 
Hausherr, I. (1955), Direction spirituelle en Orient autrefois, Roma, Pontificium Institutum Orientalium Studiorum.

Horton, R. (1967), "African Traditional Thought and Western Science", Africa. Journal of the International African Institute, 37/1, p. 50-71 et 37/2, p. 155-187.

James, W. (1999 [1902]), The Varieties of Religious Experience. A Study in Human Nature, New York, Modern Library.

Jenkins, C. (1907-1909), "Origen on I Corinthians ", The Journal of Theological Studies, 9, p. 231-247, 333-372, 500-514 et 10, p. 29-51.

Koselleck, R. (1997 [allemand 1986]), "Histoire sociale et histoire des concepts ", dans L'expérience de l'histoire, Paris, Gallimard, p. 101-119. (1997b), "The Temporalization of Concepts ", dans Finnish Yearbook of political Thought, 1, p. 16-24.

Lampe, G. W. H. (1968), A Patristic Greek Lexicon, Oxford, Oxford University Press.

Larchet, J.-Cl. (1997), Thérapeutique des maladies spirituelles, Paris, Cerf.

Levko, J. (1996), "The Relationship of Prayer to Discretion and Spiritual Direction for John Cassian ", St. Vladimir's Theological Quaterly, 40, p. 155-171.

Liddell, H. et al. (19969), A Greek-English Lexicon. Oxford, Clarendon Press.

Lienhard, J. T. (1982), "Discernment of Spirits" in the Early Church", dans E. A. Livingstone, dir., Studia Patristica, Vol. XVII. Papers Presented at the Eighth International Conference on Patristic Studies, Oxford, Sept. 3-8, 1979, II, Oxford/New York, Pergamon Press, p. 519522.

MacMullen R. (2011 [anglais 1996]), Christianisme et paganisme du IV au VIII siècle, Paris, Perrin.

Malinowski, B. (1925), "Magic, Science and Religion", dans J. Needham, Science, Religion and Reality, New York, Macmillan.

Marrou, H.-I. (1977), Décadence romaine ou antiquité tardive?, Paris, Seuil.

Martin, R. P. (1992), "Gifts, spiritual», dans D.N. Freedman, dir., The Anchor Bible Dictionary, Vol. 2, New York, Doubleday, p. 1015-1088.

Marty, F. (1958), «Le discernement des esprits dans le Perì archon», Revue d'ascétique et de mystique, 34, p. 153-164. 
Meier, H. G. (1971), «Begriffsgeschichte», dans J. RitTer, dir., Historisches Wörterbuch der Philosophie, vol. I, Basel, Schwabe Verlag, col. 788808 .

Merkel, H.et Wallis, R. (1999), "Charisma », dans E. Fahlbusch et al., The Encyclopedia of Christianity, Vol. 1, Grand Rapids/Cambridge/ Leiden/Boston/Köln, p. 402-404.

Miquel, P. (1997), Mystique et discernement, Paris, Beauchesne.

Monaci Castagno, A. (2009), "Il "discernimento degli spiriti" in Origene », Rivista di Storia del Cristianesimo, 6, p. 9-20.

Moreschini, C. et Norelli, E. (2000, [italien 1995]), Histoire de la littérature chrétienne ancienne grecque et latine. 1. De Paul à l'ère de Constantin, Genève, Labor et Fides.

Отто, R. (1995) [allemand 1917]), Le sacré, Paris, Payot.

Painchaud, L. et Wees, J. (2002), "Connaître la différence entre les hommes mauvais et les bons: le charisme de clairvoyance d'Adam et Ève à Pachôme et Théodore ", dans H. B. Bethge et al., dir., For the Children, Perfect Instruction. Studies in Honor of Hans-Martin Schenke, Leiden/Boston, Brill, p. 139-155.

Pétrement, S. (1947), Le dualisme chez Platon, les gnostiques et les manichéens, Paris, Presses Universitaires de France.

Puech, H.C. (1979), «Péché et confession dans le manichéisme (1965) », Sur le manichéisme et autres essais, Paris, Flammarion, p. 169-178.

Regnault, L. (1990), La vie quotidienne des Pères du désert en Égypte au IV siècle, Paris, Hachette.

Rich, A. D. (2007), Discernment in the Desert Fathers. Diakrisis in the Life and Thought of Early Egyptian Monasticism, Milton Keynes, Paternoster.

Rubenson, S. (1990), The Letters of St. Antony. Origenist Theology, Monastic Tradition and the Making of a Saint, Lund, Lund University Press.

(1999), "Origen in the Egyptian Monastic Tradition of the Fourth Century ", dans W. A. Bienert et U. Kühneweg, dir., Origeniana Septima, Leuven, Leuven University Press, p. 319-337.

(2011), "Antony and Ammonas. Conflicting or Common Tradition in Early Egyptian Monasticism?", dans D. Bumazhnov et al., Bibel, Bizanz und Christlicher Orient. Festschrift für Stephen Gerö zum 65. Geburstag, Leuven, Peeters, p. 185-201. 
Salamito, J.-M. (1995), "La christianisation et les nouvelles règles sociales ", dans J. MAYEUR et al.., Histoire du christianisme 2, Naissance d'une chrétienté (250-430), Paris, Desclée, p. 675-717.

Schmitт, C. (1988 [allemand 1922]), Théologie politique, Paris, Gallimard.

Therrien, G. (1973), Le discernement dans les écrits pauliniens, Paris, Gabalda.

Vecoli, F. (2003), "Verso il conflitto tra pagani e cristiani nell'alto Egitto tra IV e V secolo", dans: Annali di Storia dell'Esegesi 20, p. 369-382.

(2006), Lo Spirito soffia nel deserto. Carismi, discernimento e autorità nel monachesimo egiziano antico, Brescia, Morcelliana.

(2007), Il sole e il fango. Puro e impuro tra i Padri del deserto, Roma, Edizioni di Storia e Letteratura.

(2009), «Trasformazione del discernimento in pratica istituzionale nella tradizione egiziana ", Rivista di Storia del Cristianesimo, 6, p. 21-41.

Von Rad, G. (1971, [allemand 1970]), Israël et la sagesse, Genève/Paris, Labor et Fides.

WaAijman, K. (1997), «Discernment. Its History and Meaning ", Studies of Spirituality, 7, p. 5-41.

\section{Résumé}

L'article analyse le discernement spirituel dans le christianisme ancien. Le but est de comprendre l'évolution conceptuelle et les fonctions religieuses de cette capacité considérée comme divine par les auteurs qui en développent la théorie. Après une première mention dans les épîtres de Paul, on constate un vide dans la théologie des premiers siècles. C'est donc seulement au $\mathrm{IV}^{\mathrm{e}}$ siècle, avec l'essor du monachisme, que le discernement spirituel acquiert toute son importance. Il représente l'instrument cognitif qui rend possible le processus décisionnel dans une situation d'ignorance: ce don permet de voir les mécanismes cachés du réel afin de toujours prendre la bonne décision. D'abord utilisé individuellement comme guide dans la pratique de l'ascèse, il finit par devenir l'emblème d'une autorité charismatique puissante et le signe de reconnaissance de la sainteté. 


\section{Abstract}

This article focuses on the spiritual discernment in ancient Christianity, in the attempt to understand the conceptual evolution and the religious functions of this capacity, considered to be divine by those authors who elaborate on its theory. After a first appearance in Paul's epistles, it is near-absent in the theology of the first centuries. Only in the IV $V^{\text {th }}$ century, with the growth of early monasticism, spiritual discernment becomes particularly significant. It comes to represent the cognitive tool that makes the decision-making process achievable in a situation of ignorance: this gift allows to see the hidden mechanisms of reality so to always make the right decision. Firstly used as a personal source of guidance in asceticism, it eventually becomes the mark of a powerful charismatic authority and the godly acknowledgement of holiness. 